\title{
Principle Analysis and Improved Design of a Solid State Power Amplifier Module
}

\author{
Xiao Wang, Gang Cheng, Shuai Liu \\ China Satellite Maritime Tracking and Control Department, Jiangyin, 214431, China
}

Keywords: Solid state amplifier; balanced amplification; MOSFET; fault analysis.

\begin{abstract}
The application of solid-state power amplifier in the transmitter has been more and more extensive. This paper analyzes the working principle of a balanced-type solid-state amplifier module based on GaN, and analyzes its common failure mechanism. Design, for most of the solid-state power amplifier module (including LDMOS tube amplifier) the design and use of a certain reference to the significance.
\end{abstract}

\section{Introduction}

At present, the measurement vehicles have been equipped with USB, UXB transmitter and the new installed UCB transmitter are solid-state power amplifier transmitter, solid-state power amplifier with a fast start-up, good maintenance, small size, light weight, high reliability Features. The core part of the transmitter solid state power amplifier module also has high gain, high linearity, good consistency, and easy interchangeability and maintenance advantages. But the solid-state power amplifier is also a high cost, high profit equipment, a single amplifier module market price should be tens of thousands of dollars, with the increase in usage, solid-state power amplifier module also appears with the various failures, the previous power amplifier maintenance mainly rely on agents This paper analyzes the working principle and common failure mechanism of the solid-state power amplifier module, and puts forward the corresponding improvement design to improve the reliability of the solid-state power amplifier and reduce the cost of equipment use. The

\section{Principle Analysis of Solid State Amplifier Module}

At present, the amplifiers used in the solid-state power amplifier modules are divided into two types: one is based on the common MOS field effect transistor and the other is the SiN-based LDMOS tube. This article to analyze the solid-state power amplifier module belongs to the first, power amplifier module is mainly composed of two parts, one power amplifier circuit, the second is the power supply control circuit, as shown in Figure 1 for the amplifier module internal circuit physical map.

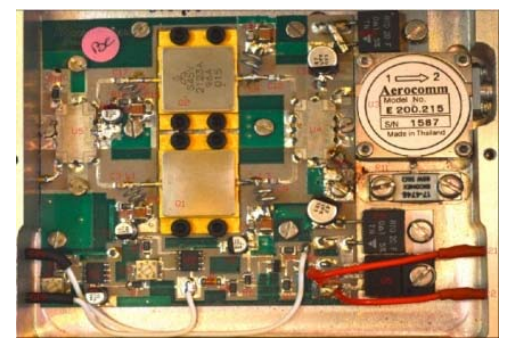

Figure 1. Power amplifier module internal circuit physical map

The power amplifier circuit is a quadrature balanced amplifier circuit composed of MOS transistor amplifier (Q1, Q2), balance bridge (U4, U5), isolator (U3), and its power is $60 \mathrm{~W}$ and gain is $12 \mathrm{~dB}$. The principle of quadrature-balanced amplifier circuit is to balance the bridge by the balance of the amplifier signal distribution and synthesis, so as to eliminate the ideal state of the impedance matching generated by the input reflection power, so that the amplifier output maximum power. When the two amplifiers are in phase and the synthesizer is ideal, the balanced amplifier gain should be the same as the one-way amplifier gain. Balanced amplifier with high stability and reliability, even if one of the circuit failure, the amplifier derating can still work. 
One of the MOS tube amplifier is based on GaN N-channel depletion-mode FET amplifier. Which belongs to the push-pull amplifier, the circuit with two transistors constitute a amplifier circuit, two transistors were amplified input signal positive half week and negative half weeks, that is, Class B amplifier, that is, with a transistor to amplify the signal half a week, As shown in Figure 2, with the other transistor to amplify the negative half of the signal, the two transistor output half-cycle signal on the amplifier load to get a complete cycle of the output signal. Push-pull amplifier circuit, a transistor work in the conduction, amplification state, the other transistor in the cut-off state, when the input signal changes to another half-week, the original conduction, amplification of the transistor into the cut-off, and the original cut off Of the transistor into the conduction, amplification of the state, two transistors continue to alternately turn on the amplification and cut-off changes.

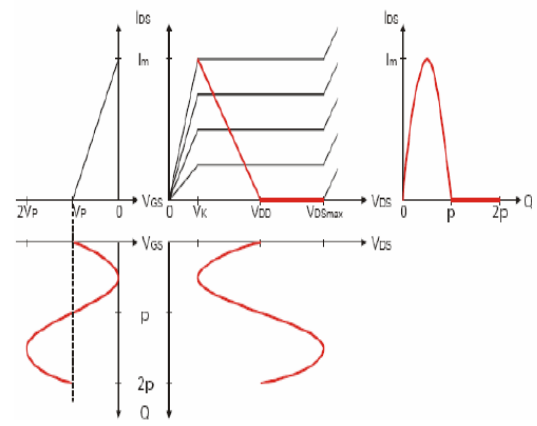

Figure 2. Class B amplifier characteristic curve

The balanced bridge is a $3 \mathrm{~dB}$ branch line coupler with a phase shift of $90^{\circ}$, and its signal transmission characteristics are shown in Fig.

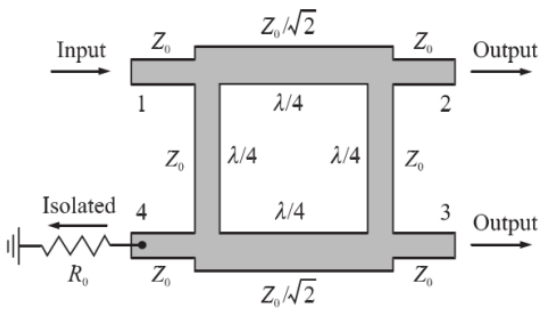

Figure 3. $3 \mathrm{~dB}$ branch line coupler

It is not difficult to see from the above, $3 \mathrm{~dB}$ bridge has a high degree of isolation, 1-port input signal is $3 \mathrm{~dB}$ bridge evenly assigned to 2,3 ports, and 2-port output signal phase will be advanced 3port $90^{\circ}$. At the same time because the characteristics of the two amplifiers are basically the same, the two amplifiers reflect the signal amplitude is basically the same, the reflection signal through the $3 \mathrm{~dB}$ bridge will be 4-port synthesis, and power resistor R0 absorption, and almost no output in the 1 port. Similarly, the balance of the bridge input and output swaps, can be used for power synthesis.

Power amplifier module power supply control circuit is composed of switching MOS tube, transistor, Zener diode and the corresponding capacitor resistor switch circuit.

\section{Analysis of Common Fault Mechanism}

Common fault of the amplifier module is divided into two main categories: one is the amplifier circuit failure, one is the power supply control circuit failure.

\subsection{Power Amplifier Circuit Failure}

There are three main types of amplifier circuit failure:

\section{A). Power amplifier burned}

The burner burner has always been the most common fault. The relationship between the drainsource current and the gate-source voltage of the N-channel depletion mode field amplifier is shown in Fig. 4, and the gate threshold voltage decreases as the temperature increases. And the current supply of the power amplifier tube gate voltage is fixed, when the amplifier tube temperature or gate ground short circuit, are likely to cause leakage current is too large, and then burn the power amplifier. As can be seen from Table 1, the highest failure rate before 2011, because the initial shipment due to the 
engine room tube fan and power amplifier box duct design unreasonable, resulting in high temperature and burn the power amplifier tube. 2013,2016 two years is also due to the implementation of multi-turn of the 921 task, the device power longer working hours, which led to increased power amplifier failure rate.

Judge the main method of power amplifier failure is: If the power amplifier circuit circuit is not open in the open state, you can determine the damage to the amplifier tube, the general failure voltage $12 \mathrm{~V}$. In the measurement or replacement of the amplifier tube should pay attention to anti-static, in the measurement of the gate voltage should pay attention to anti-ground.

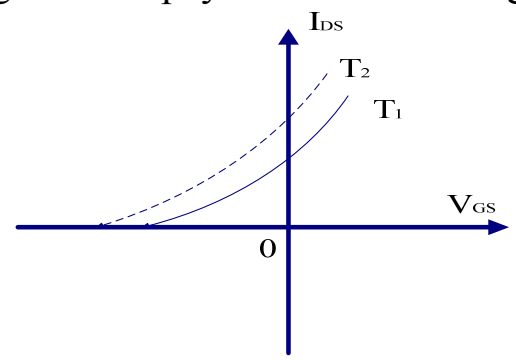

Figure 4. The relationship between the leakage current and the gate - source voltage of the amplifier B). The Drain supply current limiting resistor burned

As shown in Fig. 4, when the current flowing through the power amplifier tube is too large, the current limiting resistor R9 or R12 on the drain power supply line is burned due to excessive heat generation, resulting in interruption of the power supply drain. If R9 or R12 burned into a short circuit state, it will lead to loss of current limiting effect, which may lead to burner burned. If the power amplifier tube short-circuit, it will lead to excessive current burnout current limiting resistance.

C). Power load burned

Absorption of the balance of the bridge U4 power after the synthesis of the reflected power of the power load R10 if over power and other reasons and burned, will lead to balanced bridge U4 input reflection power is large, and then easy to burn the power amplifier. Current limiting resistors, power loads and amplifier failures are often associated, and after one of the links fails, check that the other links are normal.

\subsection{Power Supply Control Circuit Failure}

Power supply control circuit failure, including transistor fault and switch MOS tube failure two cases. Whether it is transistor or switch MOS tube failure, generally will cause the switch MOS tube off, interrupt the power amplifier drain. In recent years, the failure rate of power supply control circuit is mainly related to the service life of equipment and the aging of equipment components.

To determine whether the power supply control circuit is faulty is: Under normal circumstances, the switch Mos tube Q5 input (source) output (drain) direct pressure drop of $0.48 \mathrm{~V}$, resistance is about $10 \mathrm{M} \Omega$. In the case of power can be measured by switching the MOS tube to the resistance, the transistor voltage drop to determine whether the device is normal.

\section{Design Improvement of Power Amplifier Module}

\subsection{Improved Balance Bridge Rear End Matching Power Resistance}

The original balance of the bridge U4 back-end power load R10 design is $10 \mathrm{~W}$ chip power load, the load design there are two drawbacks: 1. Chip-type load welding process requirements, in the heat easily lead to Weld. 2. According to the previous balance bridge transmission characteristics analysis, if the power amplifier are working properly, then the $10 \mathrm{~W}$ load to meet the basic requirements of the absorption of reflected power, if the power amplifier failure, full power work, single amplifier power will reach $50 \mathrm{~W}$ or more, At this point the power load will absorb a larger reflection power, which due to overpower caused by R10 burned, resulting in excessive reflection of the power amplifier tube burned. In response to this design problem, the R10 to $50 \Omega 60 \mathrm{~W}$ lead-type power resistance, after practice tests show that greatly improved the reliability of the circuit in recent years greatly reduced the failure rate. 


\subsection{Improved Module Power Supply Control Circuit}

The current module power supply control circuit is composed of transistor and MOS tube together constitute the switching circuit, responsible for the amplifier tube drain voltage power supply, power amplifier gate voltage is often power supply, in high temperature, the transistor and MOS tube reliability will be reduced, If the transistor or MOS tube in any part of the failure, can not guarantee the normal power amplifier drain, while intensive patch circuit to increase the complexity of the electromagnetic environment and maintenance of the difficulty. In the improved power amplifier module design, the use of monolithic TTL driver instead of the entire power supply control circuit. The current single-chip drive excellent performance, can improve the reliability of the circuit and maintenance of convenience, is conducive to improving the internal electromagnetic environment within the module to improve the electromagnetic spectrum purity.

\subsection{Increase the Temperature Compensation Circuit}

In the improved design, the temperature compensation circuit is added to control the gate supply voltage in real time to realize the stable control of the amplifier current, reduce the loss of the power amplifier tube and improve the life and reliability of the power amplifier tube, as shown in Fig. 5 The power supply control circuit is replaced with the ADG1419 driver. It should be noted that the temperature compensation circuit in the LDMOS tube amplifier circuit than the gate of the negative pressure of the GaN amplifier tube easier to design and application.

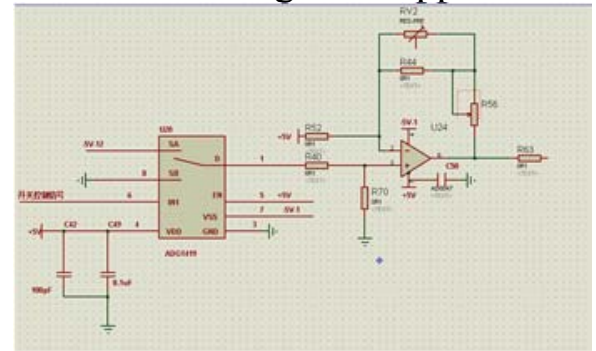

Figure 5. Temperature compensation circuit schematic

\section{Summary}

Although the current application of the amplifier module design has its own characteristics, but the actual working principle are similar, this paper analyzes the principle of the amplifier module and the proposed design can also be analogous to the current use of solid-state power station, help to reduce equipment maintenance Cost, to carry out the use of power amplifier equipment maintenance and theoretical research has a guiding significance.

\section{References}

[1]. Liao S.Microwave Devices and Circuit- s [M].Prentie-Hall 1990; 3ed:154-156.

[2]. Chong T, Rendava S.Design and Performance of a 1.6-2.2GHz Low-Noise, High Gain Dual Amplifier in GaAs E-pHEMT[C] // Microwave Conference Proeedings, 2005.APMC2005.Asia$\mathrm{Pa}$-cific Conference Proceedings, Volume, 2005, 4 (3):4-7. 